\title{
Esophageal Cancer in Korea: Epidemiology and Treatment Patterns
}

\author{
Seong Yong Park, M.D., Ph.D., Dae Joon Kim, M.D., Ph.D. \\ Department of Thoracic and Cardiovascular Surgery, Severance Hospital, Yonsei University College of Medicine, Seoul, Korea
}

\author{
ARTICLE INFO \\ Received May 4, 2021 \\ Revised July 3, 2021 \\ Accepted July 16, 2021 \\ Corresponding author \\ Seong Yong Park \\ Tel $82-2-2228-2140$ \\ Fax 82-2-393-6012 \\ E-mail syparkcs@yuhs.ac \\ ORCID \\ https://orcid.org/0000-0002-5180-3853
}

\begin{abstract}
According to statistics from 2017, esophageal cancer is the fifteenth most common cancer and the eleventh most common cause of cancer-related death in Korea. The most common pathology is esophageal squamous cell carcinoma. Moreover, the incidence of esophageal cancer has been gradually decreasing in Korea, and the percentage of early-stage cases has gradually increased to the point that it is higher than that of other countries. The 5-year relative survival rate has improved over time. Approximately 800 esophagectomy procedures are performed annually. Using a cut-off number of 21 cases per 2 years to define high-volume centers, it was found that $70 \%$ of esophagectomies were performed by a few high-volume centers. Unfortunately, there is no nationwide registry or database on esophageal cancer and esophagectomy in Korea. Efforts to establish a nationwide database on esophageal cancer and esophagectomy should be made.
\end{abstract}

Keywords: Esophageal cancer, Epidemiology, Statistics, Surgery

\section{Introduction}

Esophageal cancer is the seventh most common cancer (604,000 new cases) and the sixth most common cause of cancer-related death (544,000 deaths) worldwide, accounting for 1 in every 18 cancer-related deaths in 2020 [1]. In both sexes, esophageal cancer is most prevalent in East Asia, followed by Southern Africa, East Africa, and Northern Europe. The epidemiology of esophageal cancer varies according to geography and pathology. The most common pathology is squamous cell carcinoma in East Asia and adenocarcinoma in Western countries. The incidence of esophageal squamous cell carcinoma (ESCC) has decreased or remained stable in Asian countries, whereas the incidence of adenocarcinoma has increased rapidly in Western countries [2]. Due to differences in epidemiology among different regions and nations, the global cancer statistics from GLOBOCAN, a project of the International Agency for Research on Cancer, do not accurately reflect the Korean situation. This brief review summarizes the epidemiology and treatment patterns of esophageal cancer in South Korea.

\section{Cancer statistics in Korea based on the Korea Central Cancer Registry}

The Korea National Cancer Incidence Database (KNCI $\mathrm{DB}$ ) is a national, population-based registry of cancer development that includes information on patients diagnosed in more than 200 hospitals in Korea, combined with data provided by central and 11 regional cancer registries that recorded information on the patients missing from other databases [3]. Since 1999, the Korea Central Cancer Registry (KCCR) has compiled the KNCI DB and reported related nationwide statistics. According to this report, the number of newly diagnosed cancer cases and cancer-related deaths was 232,255 in 2017 (age-standardized rate, 264.4/100,000) and 78,863 (age-standardized rate, $76.6 / 100,000)$, respectively. The overall cancer incidence rates increased annually by $3.5 \%$ from 1999 to 2011 and decreased annually by $2.7 \%$ after 2011 . Cancer-related deaths have decreased since 2002 by $2.8 \%$ annually. The 5 -year relative survival rate for all cancer patients between 2013 and 2017 was $70.4 \%$ [3].

Fig. 1 shows the incidence and cancer-related deaths of various cancers in Korea in 2017. There were 2,483 cases of

Copyright (C) 2021, The Korean Society for Thoracic and Cardiovascular Surgery

(i) \$ This is an Open Access article distributed under the terms of the Creative Commons Attribution Non-Commercial License (http://creativecommons.org/licenses/ by-nc/4.0) which permits unrestricted non-commercial use, distribution, and reproduction in any medium, provided the original work is properly cited. 
A

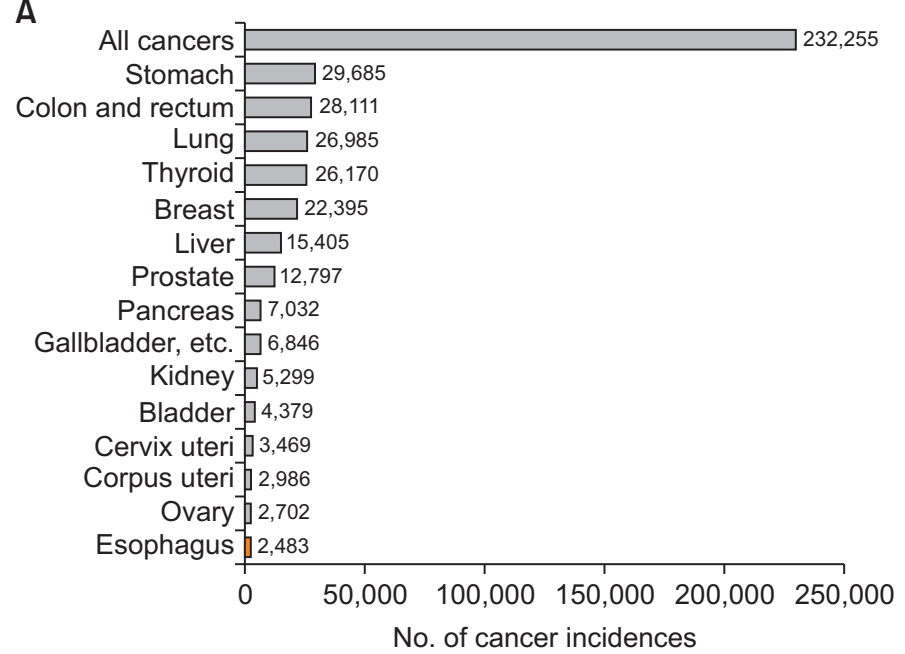

B

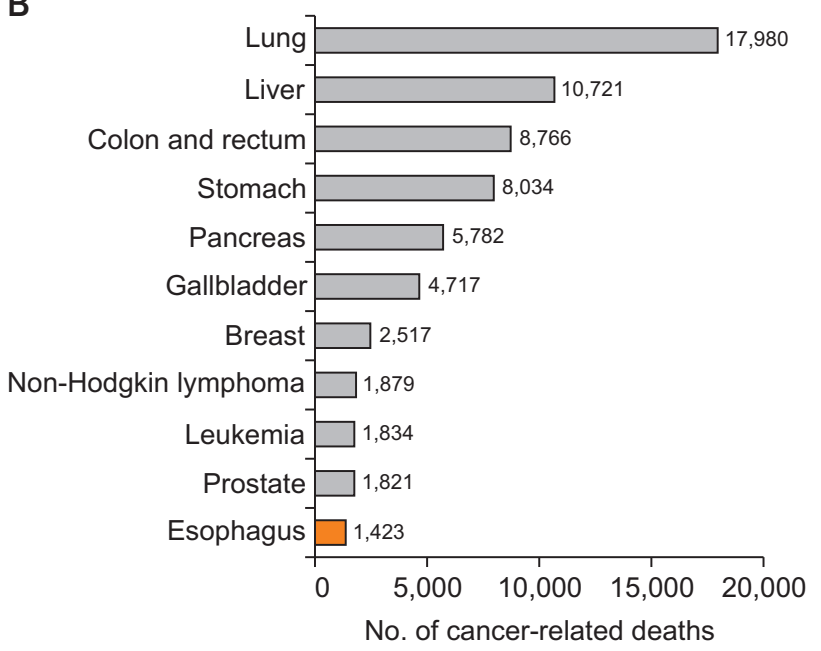

Fig. 1. Cancer incidence (A) and deaths (B) in Korea, 2017.

A

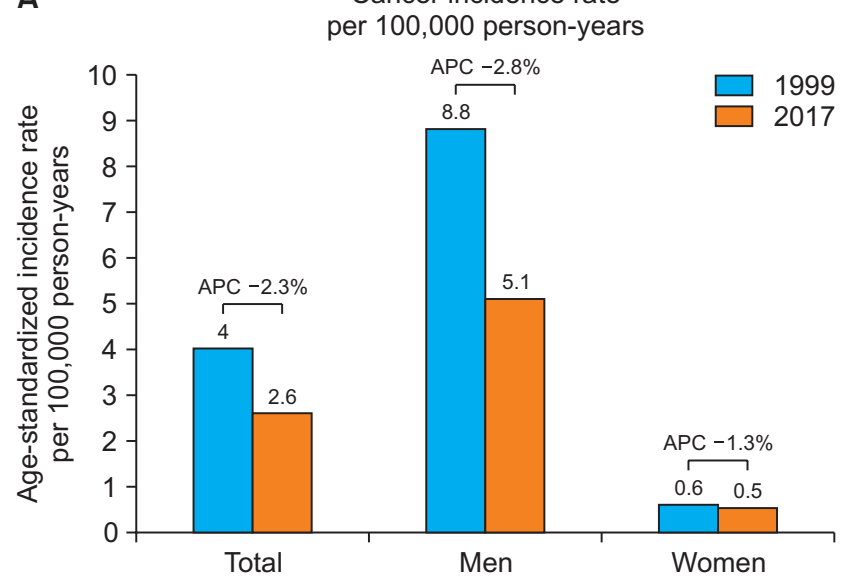

B

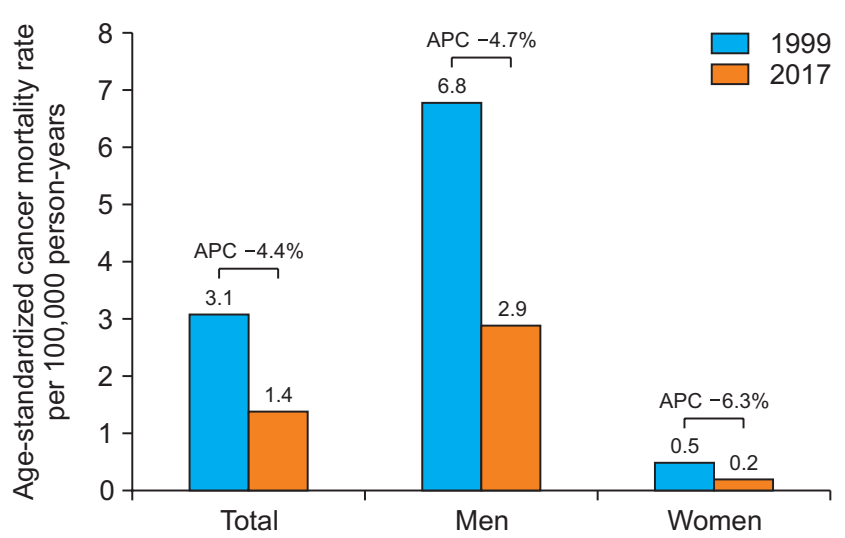

Fig. 2. Trends in the age-standardized incidence rate (A) and cancer mortality (B) per 100,000 person-years between 1999 and 2017. APC, annual percentage change.

esophageal cancer, accounting for $1.0 \%$ of all cancers. In 2017, esophageal cancer was the fifteenth most common cancer and the eleventh most common cause of cancer-related death in Korea. The crude incidence rate per 100,000 person-years of esophageal cancer was 4.8 (men, 8.8; women, 1.0), and the age-standardized incidence rate per 100,000 person-years was 2.6 (men, 5.1 ; women, 0.5 ). Comparing data from 1999 and 2017, the age-standardized incidence rate per 100,000 person-years has significantly decreased (Fig. 2A) [4]. The male-to-female ratio was 10.2:1. Esophageal cancer was most prevalent among patients in their 60s (33.7\%), followed by those in their 70s (29.8\%). The most common pathology was squamous cell carcinoma, which accounted for $90.2 \%$ of cases, followed by adenocarcinoma (3.1\%). The KNCI DB collected staging data using the summary staging system (localized, regional, and distant stages) developed under the Surveillance, Epidemiology, and End Results (SEER) program. The proportion of localized and regional cancers was higher than the proportion of distant cancers (Fig. 3). This may be related to the National Screening Program, which involves performing esophagogastroduodenoscopy as a screening test. In Korea, the incidence of gastroesophageal reflux disease and obesity is currently increasing. As a result, the incidence of esophageal adenocarcinoma or junction cancer may also increase, although the incidence of these cancers in Korea has not changed since the 1990s [5]. According to GLOBOCAN 2020, the age-standardized incidence rate per 100,000 person-years in East Asia was 18.2 in men and 6.8 in women. Compared to other countries in East Asia, 
the incidence of esophageal cancer in Korea is lower than that in Japan and China, and the age-standardized incidence and death rates in Korea are half of those registered in China [6]. However, the exact reasons have not yet been elucidated.

The crude and age-standardized cancer mortality rates of esophageal cancer in 2017 were 2.8 (men, 5.0; women, 0.5 ) and 1.4 (men, 2.9; women, 0.2) per 100,000 person-years, respectively. Age-standardized cancer mortality has also decreased over the past 20 years (Fig. 2B). The 5 -year relative survival of ESCC has increased gradually, from 14\% (1993-1995) to 38\% (2013-2017) (Fig. 4). The 1-, 3 -, and 5-year relative survival rates (\%) of esophageal can-

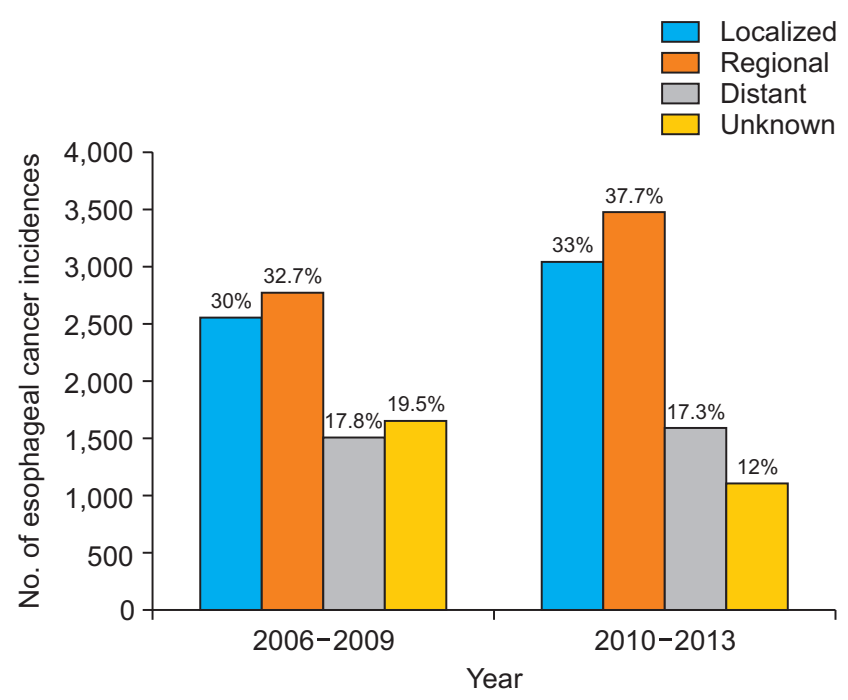

Fig. 3. Incidence rates of esophageal cancer per 100,000 person-years according to the Surveillance, Epidemiology, and End Results (SEER) staging system. cer from the time of diagnosis, according to the SEER stage, have decreased over the past 20 years (Fig. 5).

\section{Nationwide cohort study}

Jung et al. [7] conducted a cohort study that analyzed data obtained by sampling tertiary hospitals in each region in Korea. Hence, the findings of Jung et al. [7] are representative of current practices regarding esophageal cancer in Korea. This retrospective cancer cohort included newly diagnosed cases of esophageal cancer at 19 tertiary hospitals and analyzed 6,354 patients between January 1, 2005, and December 31, 2017. These data accounted for $25.8 \%$ of esophageal cancer cases among KCCR registrants in South Korea. The mean age of the patients was $64.9 \pm 9.0$ years, and $92.9 \%$ were men. Esophageal cancer was diagnosed between the ages of 25 and 98 years and predominated in patients aged 60-69 years. The most common pathology was squamous cell carcinoma (96.9\%). In contrast, adenocarcinoma (3.1\%) made up only a small proportion. These results are similar to those obtained from the KCCR data.

As described earlier, the KCCR database uses the SEER staging system, meaning that detailed information on staging, treatments, and clinical outcomes is not available. On the contrary, the cohort study introduced above provided detailed information on the treatment of esophageal cancer in Korea. Clinical stage I disease accounted for 30\% of all esophageal cancers, which is a higher proportion than has been published in studies from Western countries, as exemplified by the proportions of $17.5 \%$ reported by the Mayo Clinic and $4.9 \%$ in a prospective multicenter UK study [8]. Regarding treatment modalities, almost all patients (93\%) received treatment, including palliative care.

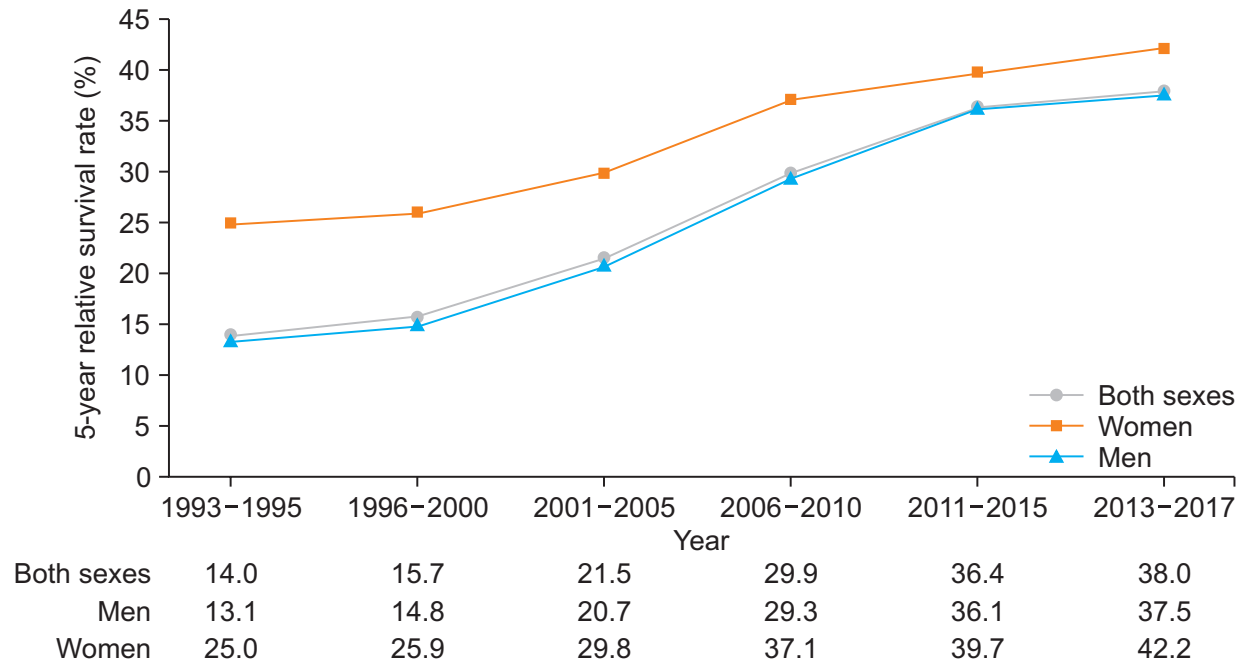

Fig. 4. Trends in the 5-year relative survival rate $(\%)$ in Korea. 

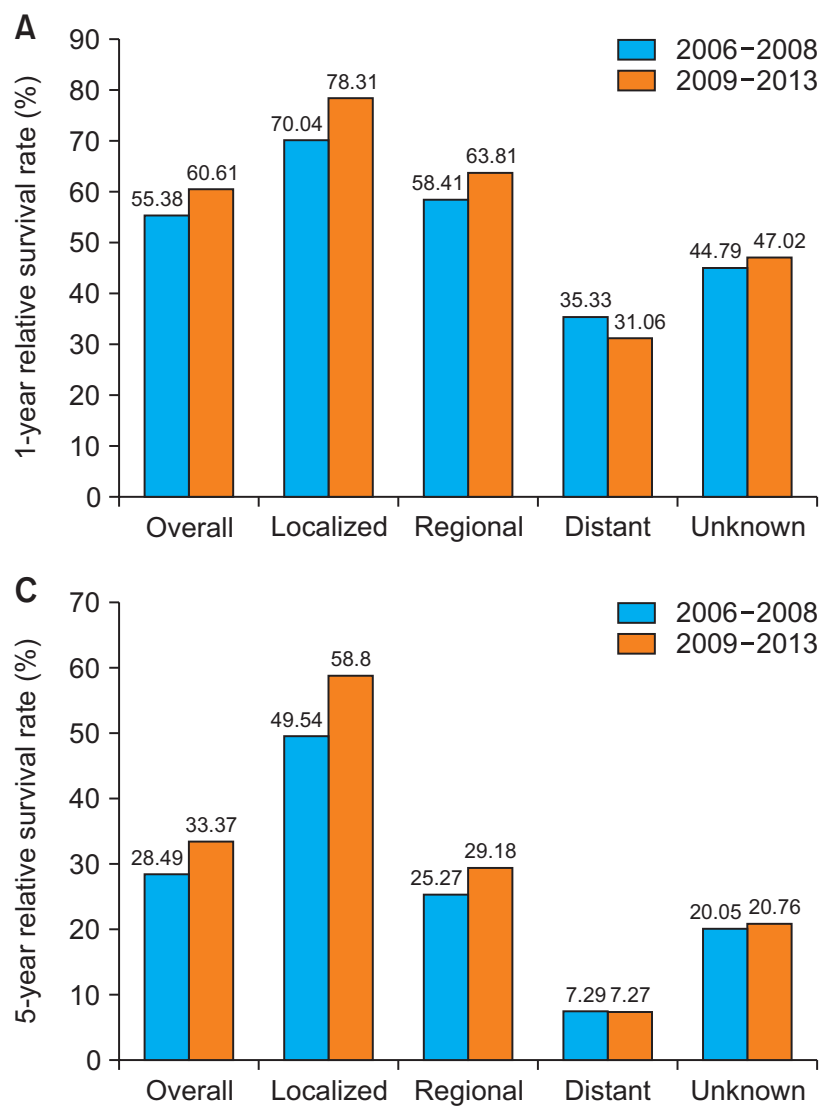

Among all the cases, 52.3\% involved surgical resection, including the Ivor Lewis operation (67.9\%), the McKeown operation (17.9\%), trans-hiatal esophagectomy (2.4\%), left thoracoabdominal esophagogastrostomy $(0.6 \%)$, and other types of surgery (11.3\%). The treatment modalities included surgery alone (31.1\%), endoscopic resection (5.8\%), neoadjuvant therapy (12.4\%), adjuvant therapy (11.1\%), and definitive chemoradiation therapy (CCRTx, 27.0\%).

Among the 1,923 patients who had clinically early-stage disease (T1N0), surgical resection was performed in 57.0\%, and endoscopic resection (including endoscopic mucosal resection and endoscopic submucosal dissection) was performed in $16.2 \%$. In patients with mucosal esophageal cancer (T1a), esophagectomy was performed in 233 patients (60.8\%), followed by endoscopic resection (31.8\%), and definitive CCRTx (7.3\%). Among the 3,510 patients with locally advanced (stage II-III) disease, definitive CCRTx was performed in $31.7 \%$, and surgery with or without combined therapy was performed in $54.5 \%$, including neoadjuvant therapy (609 patients), adjuvant therapy (518 patients), and surgery alone (788 patients). The 5 -year overall survival rates were $45.7 \%, 72.63 \%, 48.1 \%, 29.9 \%$, and $16.6 \%$ in stages I, II, III, and IV, respectively.

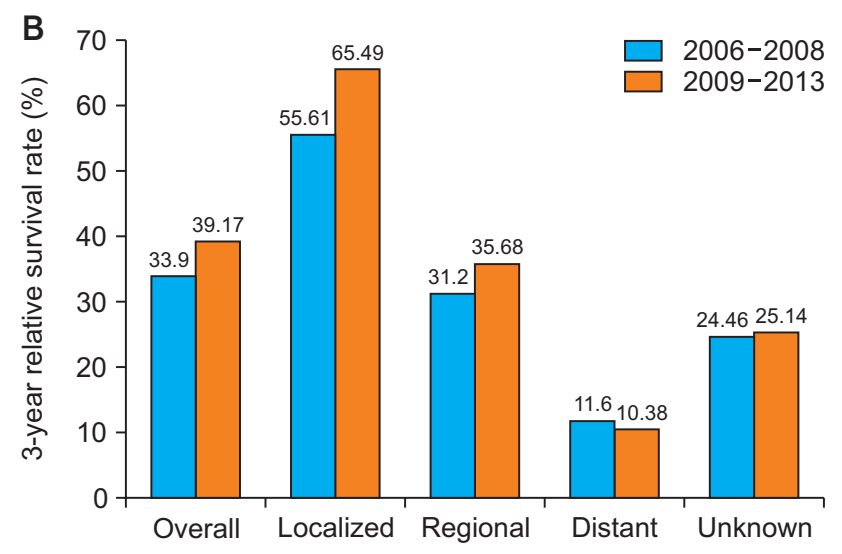

Fig. 5. (A-C) The 1-, 3-, and 5-year relative survival rates (\%) of patients with esophageal cancer from the time of diagnosis, according to the SEER (Surveillance, Epidemiology, and End Results) staging system.

\section{Analysis of surgical treatment based on the Health Insurance Review \& Assessment Service data}

Medical treatment for all Korean citizens is supported by the National Health Insurance System, and all medical practices are evaluated by the Health Insurance Review \& Assessment Service (HIRA), which is a sub-organization of the National Health Insurance System. The HIRA reported the relationship between hospital volume and several surgical outcomes, including esophagectomy in Korean hospitals, based on 2007-2013 data from the National Health Insurance System.

In 2007, the HIRA reported an analysis of esophagectomy cases performed between 2005 and 2006. In the span of 2 years, 1,272 patients underwent esophagectomy for esophageal cancer, and the in-hospital mortality rate was $3.38 \%$. Complications were defined as medical complications (cardiovascular complications, pulmonary complications, renal failure, and/or hepatic failure) and surgery-related complications (infection, bleeding, wound dehiscence, and/or reoperation). The overall complication rate was $65.17 \%$. Age, emergency admission, Charlson comorbidity 
index score, and hospital type (teaching hospital or not) were related to operative mortality. The minimum requirement for the surgical volume to show acceptable mortality was calculated as 21 cases per 2 years, which was used as the cut-off value to define higher-volume centers. Higher-volume centers showed lower operative mortality $(2.07 \%$ versus $6.20 \%)$, lower overall complication rates $(62.95 \%$ versus $69.98 \%$ ), and shorter hospital stays (26.30 days versus 30.55 days) than in lower-volume hospitals. Thirteen hospitals (17.1\%) were higher-volume centers, and they performed 869 esophagectomies (68.2\%) between 2005 and 2006. This was the first report on the volume-outcome relationship of esophagectomy in Korea. According to this report, high-volume centers are expected to show better postoperative outcomes.

In 2013, the HIRA published a report on esophagectomies performed between 2011 and 2012. The number of esophagectomies was 1,751, and 2-field approaches were performed in 1,214 patients (69.3\%). The operative mortality rate was $4.68 \%$. From this report, it can be assumed that approximately 800-900 procedures were performed annually during this period. The cut-off value used to define a high-volume center was the same as in previous reports (21 cases per 2 years). The operative outcomes were also consistent with those from the previous report. High-volume centers showed lower operative mortality $(3.26 \%$ versus $8.68 \%$ ), shorter hospital stays (25.68 days versus 37.97 days), and lower costs $(16,166,000$ Korean won [KRW] versus 20,060,000 KRW). Sixteen hospitals (19.5\%) had high-volume centers, and they performed 1,290 esophagectomies (73.7\%) over 2 years. Among the 16 hospitals with high-volume centers, 14 showed an operative mortality rate lower than $5 \%$, and these hospitals were located mainly in the Seoul metropolitan area. The proportion of high-volume centers was lowest for esophagectomy (16 hospitals, 19.5\%) in comparison with other surgical procedures such as gastrectomy, pancreatic cancer surgery, and liver cancer surgery.

The HIRA data revealed the current status of esophagectomy in Korea. However, these data have limitations. The HIRA only analyzed esophagectomy covered by the $\mathrm{Na}$ tional Health Insurance System. Robotic esophagectomy, which is not covered by national insurance, was not included in this analysis. Unfortunately, there is no separate registry or database for esophagectomy in Korea. Further efforts to establish a nationwide registry or database are required.

\section{Conclusion}

According to statistics from 2017, esophageal cancer is the fifteenth most common cancer and the eleventh most common cause of cancer-related death in Korea. The incidence of esophageal cancer has gradually decreased. ESCC is the most common pathology. The percentage of early-stage carcinoma in Korea is higher than that in other countries, and the proportion of esophageal cancer diagnosed at the early-stage has also increased gradually. The 5 -year survival rate has improved over time. Approximately 800 esophagectomies are performed annually. A cut-off of 21 cases per 2 years was established to define high-volume centers, and $70 \%$ of esophagectomies were performed by a few high-volume centers. Unfortunately, there is no nationwide registry or database on esophageal cancer and esophagectomy in Korea. Efforts should be made to establish a nationwide database on esophageal cancer and esophagectomy.

\section{Conflict of interest}

No potential conflict of interest relevant to this article was reported.

\section{Acknowledgments}

The authors thank Prof. Hyeon Chang Kim (Department of Preventive Medicine, Yonsei University College of Medicine) for reviewing the manuscript and Medical Illustration \& Design Team for providing excellent support with medical illustrations.

\section{ORCID}

Seong Yong Park: https://orcid.org/0000-0002-5180-3853

Dae Joon Kim: https://orcid.org/0000-0002-2182-010X

\section{References}

1. Sung H, Ferlay J, Siegel RL, et al. Global cancer statistics 2020 : GLOBOCAN estimates of incidence and mortality worldwide for 36 cancers in 185 countries. CA Cancer J Clin 2021;71:209-49.

2. Edgren G, Adami HO, Weiderpass E, Nyren O. A global assessment of the oesophageal adenocarcinoma epidemic. Gut 2013;62:1406-14.

3. Hong S, Won YJ, Park YR, et al. Cancer statistics in Korea: incidence, mortality, survival, and prevalence in 2017. Cancer Res Treat 2020;52:335-50.

4. Shin A, Won YJ, Jung HK, et al. Trends in incidence and survival of 
esophageal cancer in Korea: analysis of the Korea Central Cancer Registry Database. J Gastroenterol Hepatol 2018;33:1961-8.

5. Kim JJ. Epidemiology of gastroesophageal junction adenocarcinoma in Korea. J Gastric Cancer 2018;18:328-38.

6. Yang S, Lin S, Li N, et al. Burden, trends, and risk factors of esophageal cancer in China from 1990 to 2017: an up-to-date overview and comparison with those in Japan and South Korea. J Hematol Oncol
2020;13:146

7. Jung HK, Tae CH, Lee HA, et al. Treatment pattern and overall survival in esophageal cancer during a 13-year period: a nationwide cohort study of 6,354 Korean patients. PLoS One 2020;15:e231456.

8. Sawas T, Killcoyne S, Iyer PG, et al. Identification of prognostic phenotypes of esophageal adenocarcinoma in 2 independent cohorts. Gastroenterology 2018;155:1720-8. 